\title{
Numerical study of pore-scale flow and noise of an open cell metal foam
}

\author{
Chen $\mathrm{Xu}^{1,2}$, Yijun $\mathrm{Mao}^{1,2^{*}}$, Zhiwei $\mathrm{Hu}^{1}$ \\ 1 Faculty of Engineering and the Environment, University of Southampton, SO17 1BJ Southampton, United Kingdom \\ 2 School of Energy and Power Engineering, Xi'an Jiaotong University, 710049 Xi'an, People's Republic of China
}

\begin{abstract}
This paper studies numerically the three-dimensional pore-scale flow inside a single cell structure of an open cell metal foam and its aeroacoustic features. Since the Reynolds number based on the pore diameter is very low, the Navier-Stokes equations are solved directly to simulate the unsteady pore-scale flow. The permeability and pressure drop obtained from numerical simulations are compared with existing reference results. Numerical results reveal that the flow drag of the pore-scale structure is dominated by the pressure drag which is mainly caused by the flow separation, while the friction drag is much smaller than the pressure drag despite a very high surface area-volume ratio of the metal foam. The unsteady flow separation contributes primarily to the pressure drag and causes the self-noise of the metal foam, therefore suppressing the unsteady flow separation, e.g., by optimizing the cell structure of the metal foam, would reduce the drag and aerodynamic noise. The aeroacoustic features, such as noise sources, spectra and the directivity pattern, are investigated, and the results reveal a high correlation between the noise generation and the flow separation. The direction of the maximum sound pressure for the studied cell is parallel to the flow, which is different to flow separation from a cylinder where the direction of maximum radiation is perpendicular to the flow.
\end{abstract}

\section{Keywords}

Open-cell metal foam; pore-scale flow; flow separation; flow loss; aerodynamic noise

*Corresponding author. Y.Mao@ soton.ac.uk; maoyijun@mail.xjtu.edu.cn (Yijun Mao). 


\section{Nomenclature}

$$
\begin{aligned}
& c_{0} \quad=\quad \text { sound speed } \\
& d \quad=\quad \text { equivalent diameter } \\
& d_{p} \quad=\quad \text { mean particle diameter } \\
& D \quad=\quad \text { diameter of a sphere } \\
& K=\text { permeability } \\
& L=\text { length of a cube } \\
& L_{D} \quad=\quad \text { flow drag } \\
& p \quad=\quad \text { static pressure } \\
& p_{a}^{\prime}=\text { acoustic pressure } \\
& Q_{v} \quad=\quad \text { volumetric flow rate } \\
& r \quad=\quad \text { distance between source and observer } \\
& \text { Re } \quad=\text { Reynolds number } \\
& S=\text { cross-section area of side surface } \\
& U \quad=\quad \text { superficial velocity } \\
& \varepsilon \quad=\text { porosity } \\
& \mu \quad=\quad \text { dynamic viscosity } \\
& v \quad=\quad \text { kinematic viscosity } \\
& \omega=\text { angular frequency } \\
& \Gamma=\text { skeleton surface of the metal foam }
\end{aligned}
$$




\section{Introduction}

Metal foams are multifunctional materials with specific geometric features, such as large surface area-volume ratio, narrow flow channels and high porosity, thus they usually have excellent performances for particular engineering applications. For examples, a large surface area-volume ratio can be used to enhance heat transfer in fluid systems [1, 2], narrow flow channels are beneficial to effectively absorb sound $[3,4]$, and a high porosity can be employed to alter the near-wall flow features [5, 6]. Moreover, compared with conventional porous materials, e.g. glass fibers, metal foams have advantages in high strength and corrosion-proof $[7,8]$, thus they can be potentially used in severe environments.

An application of metal foams is to control the flow and its associated aerodynamic noise. Recently, some experimental studies showed that open-cell metal foams (OCMFs) can significantly reduce the aerodynamic noise radiated from airfoils $[9,10]$, cylinders $[11]$, highspeed trains [12], aero-engines [13-15] and axial and centrifugal fans [16-18]. However, one drawback of OCMFs is that the pressure drop of the flow through OCMFs is significant, which usually affects the aerodynamic performance in a negative manner. Moreover, the experimental investigations in [18] also indicated that the structural parameters of OCMFs, such as pores per inch (PPI) and porosity, have a significant impact on the noise reduction levels. Therefore, investigating the detailed flow pattern inside the porous region of OCMFs is meaningful in order to decrease the pressure drop and to maximize the noise reduction.

However, the geometry of OCMFs is complex and the interior flow channels are usually very narrow, it is difficult to measure the unsteady flow field inside the porous region by using either intrusive or non-intrusive techniques. Therefore, only numerical simulations can be used to understand the detailed physical patterns of pore-scale flow through porous regions. 
In general, flow around and through porous regions can be simulated by using one of the following three approaches. The first method utilizes a permeable boundary condition to model the effect of the porous zone, in which the normal velocity at the permeable boundary is usually assumed to be proportional to the local static pressure. Some representative studies are listed below. Jimenez et al. [19] simulated the turbulent shear flow over a porous surface. Tinetti et al. [20] numerically studied flow passing porous vane surfaces. Lai and Luo [21] studied the flow through an a low speed cavity with porous boundaries at the cavity bottom wall and also predicted the noise radiation from the cavity. Suzuki et al. [22] investigated the flow around a porous circular cylinder. Although it is easy to consider the effect of the porous material on the flow by using the permeable boundary method, the numerical results are highly sensitive to the boundary condition, which is usually very difficult to set accurately without detailed knowledge of the flow within the porous material. Moreover, the permeable boundary method cannot provide information of the flow field inside porous regions.

The second method is the volume-averaged method, where modified Navier-Stokes equations are employed to describe the macroscopic flow in porous regions [23-25]. The momentum equation is modified by adding a source correction term, which represents the pressure drop, described by the Darcy's equation [26] or the Ergun's equation [27], of the flow in porous regions. This method has been widely utilized to study the flow characteristics of porous zones. For examples, two-dimensional laminar [28] and turbulent [29, 30] flows, as well as threedimensional laminar and turbulent flows [31] around a porous circular cylinder, two-dimensional flow [32] and three-dimensional flow [33] past a porous trailing edge. Compared with the permeable boundary method, the volume-averaged method has the capability to analyze the effect of structural parameters, such as pore diameter, porosity, on the macroscopic flow. In the 
volume-averaged method, however, the actual pore-scale flow inside the porous region is approximately represented by the macroscopic flow. This approximation is usually acceptable for analyzing the macroscopic flow around porous regions, though could cause significant deviations of unsteady pore-scale flow and associated acoustic features. For example, the volume-averaged method cannot capture the unsteady pressure fluctuation acting on solid skeletons, which highly affects the aeroacoustic radiation.

Since the above two methods are not able to reveal the detailed flow physics and to analyze the aerodynamic noise sources, we have to seek an alternative method, i.e., pore-scale simulation to resolve directly the interaction between the flow and the geometry of porous materials. Although this method is computationally expensive, it brings the benefit of detailed flow information for physical analysis. For examples, Krishann et al. [34] and Boomsma et al. [35], respectively, simulated pore-scale flow and heat transfer inside the OCMF, and the numerical results provided detailed three-dimensional velocity and temperature fields. As the flow velocity in both the above-mentioned numerical studies was very low, the steady flow was simulated by directly solving the Navier-Stokes equations.

As we know, the overall flow drag is composed of the pressure drag and friction drag. However, to the best knowledge of the authors, few studies were performed to investigate the pressure drag and friction drag of OCMFs. Krishnan et al. [34] compared the friction factor calculated from numerical simulations with the experimental data, and the numerical results in [35] plotted the pressure drop along the streamwise direction. However, neither of them compared the pressure drag and friction drag in their studies, thus it is still an open question which type of drag dominates the flow through OCMFs. In this paper, we perform the pore-scale simulations to study the three-dimensional unsteady flow and analyze the features of the pressure 
drag and the friction drag based on the pore-scale flow results obtained from numerical simulations. The aerodynamic noise generated from the flow interaction with the OCMFs is also analyzed based on the unsteady flow simulation results.

The remainder of this paper is organized as follows. Section 2 describes the cell geometry of a typical OCMF which has been used in previous experimental studies [16-18], and introduces the numerical method for simulating the pore-scale flow and then performs the numerical validation. In Section 3, detailed analysis on the pore-scale flow and the flow drag is carried out, and the aeroacoustic feature is also analyzed. Section 4 draws conclusions.

\section{Numerical methodology and validation}

\subsection{Model description}

OCMFs are composed of structures of multiple sectional cubic cells arranged periodically. It is assumed that the flow patterns in each cell of the OCMFs are the same statistically after the flow is fully developed, thus the computational domain can be limited to a single structural cell in which physical parameters on a pair of upstream and downstream boundaries can be assumed to be periodic. This approach has been previously applied to study flow in straight pipes and channels with a constant cross section, where the gradient of all flow variables, except pressure, in the streamwise direction is zero. The pressure gradient should be a non-zero constant as it is needed to drive the flow. Moreover, the concepts of fully developed flow and heat transfer have been generalized by Patanker et al. [36] to accommodate ducts whose cross-sectional area varies periodically in the streamwise direction. Examples of using this approach for flow simulation are listed as follows. Hu et al. [37, 38] directly simulated the turbulence channel flow driven by a constant pressure gradient along the streamwise direction, Kuwahara et al. [39] employed large eddy simulation (LES) to predict unsteady flow through a periodic array of square cylinders, and 
Krishann et al. [34] performed a direct numerical simulation of steady flow and heat transfer inside the OCMF. It is should be noted that this numerical treatment limits eddies considered to the length of a single cell or smaller.

Fig. 1(a) illustrates a single body-centered-cubic cell of OCMFs, in which the skeleton (shadow region) is generated by the intersection between a sphere centered at the center of the cube with a diameter $D$ and eight spheres centered at the vertexes of the cube with the same diameter. Details can be found in [34]. Therefore, the structural parameters of the OCMFs are actually determined by two variables, i.e., the length of the cube $L$ and the diameter of the sphere $D$, and the latter must be larger than the former to ensure the interaction of adjacent spheres. Moreover, the porosity of the cell can be calculated by [34]

$$
\varepsilon=\frac{\pi D^{3}-2 \pi(2 D+\sqrt{3} L / 2)(D-\sqrt{3} L / 2)^{2}-3 \pi(D-L)^{2}(2 D+L)}{3 L^{3}}
$$

Experimental results in previous studies [17, 18] have shown that the metal foams with PPIs ranging from 20 to 40 and porosity larger than $90 \%$ have good performances in controlling the flow and flow-generated noise. Therefore, the current study performs numerical simulations for the OCMF with a side length $L$ of $1 \mathrm{~mm}$ and $\mathrm{PPI} \approx 25$. The diameter $D$ is $1.02 \mathrm{~mm}$, which gives a porosity $\varepsilon=96.5 \%$ calculated from Eq. (1).

The cross-section area of each side surface can be calculated by [34]

$$
S=-D^{2} \arccos \frac{L}{D}+L \sqrt{D^{2}-L^{2}}+\frac{\pi}{4}\left[2 D^{2}-L^{2}\right]
$$

and an equivalent diameter $d$ is defined as $d=\sqrt{4 S / \pi}$. The superficial velocity $U$ through the metal foams can be calculated by $U=Q_{v} / S$, where $Q_{v}$ is the volume flow rate. Moreover, the Reynolds number based on the superficial velocity $U$ and the equivalent diameter $d$ is defined 
by $\operatorname{Re}=U d / v$, where $v$ is the kinetic viscosity. In this study, flow simulations were performed at two different Reynolds numbers, $\operatorname{Re}=130$ and 250 , respectively.

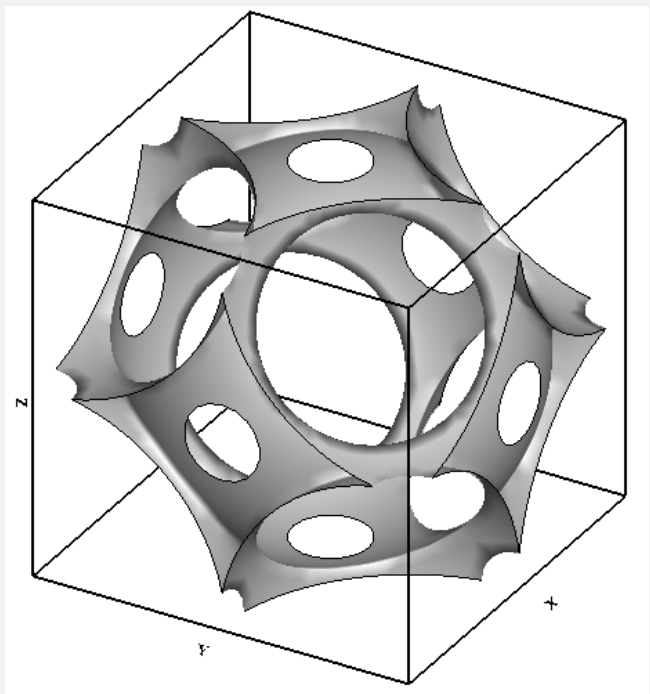

(a)

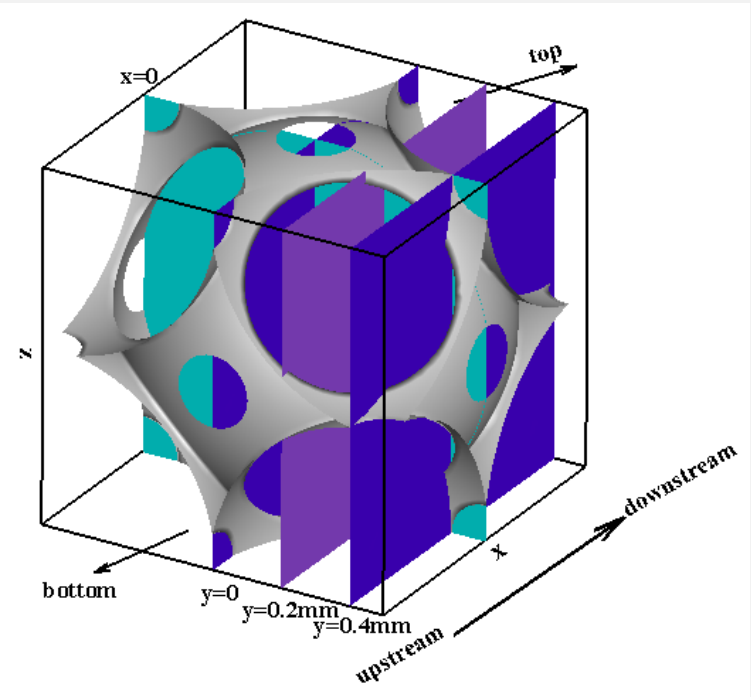

(b)

Fig. 1 Computational domain: (a) skeleton of a cell; (b) schematic of cross sections

\subsection{Numerical method}

The cell geometry consists of curved solid surfaces facing the flow at different orientations therefore flow separation is inevitable and likely to happen behind most surfaces. However, previous investigation [34] suggested that the flow at such low Reynolds numbers studied in this paper is laminar. Therefore, the Navier-Stokes equations are directly solved without using any turbulence closure model, and the finite volume method is employed to ensure that the flux is conserved through the boundaries of adjacent cells. The SIMPLE algorithm [40] is used for solving pressure-velocity coupling. Spatial discretization is performed using a second-order upwind scheme. It is known that upwind schemes are dissipative while central difference schemes are non-dissipative but dispersive. The non-dispersive nature of central difference schemes would predict unrealistic fluctuations in regions of sharp changes, such as flow separation points, which could introduce significant computational errors for predicting the 
aerodynamic noise. Therefore, the second-order upwind scheme is used in this paper. The temporal discretization follows a second-order implicit scheme.

The boundary conditions are defined below. Non-slip boundary conditions are applied at all wall surfaces, and periodic boundary conditions are used for two pairs of side surfaces shown in Fig. 1. Moreover, as the flows are assumed to be fully developed in the present simulations, periodic boundary conditions are also used for the pair of upstream and downstream boundary surfaces. The driven pressure gradient in the streamwise direction is obtained after the numerical simulation by setting a specific flow rate through the cell structure.

The direct numerical simulation requires that the meshes must be fine enough to resolve the energy-contenting structures. As shown in Fig. 2, the fluid region is discretized by tetrahedral elements with nearly equivalent size of adjacent cells, and the expansion ratio for all elements is not larger than 1.1. Five sets of computational meshes are generated with cells of $0.9 \times 10^{5}$, $1.9 \times 10^{5}, 3.4 \times 10^{5}, 7.4 \times 10^{5}$ and $10^{6}$, respectively. In the above sets of meshes, the number of uniformly distributed nodes on each side of the cubic computational domain is 56 for the finest meshes. Since no wall function is used in the present simulations, a sufficiently high near-wall grid resolution is required to accurately resolve the high shear flow in the boundary layers as well as to properly capture the flow separation. Fig. 3 illustrates the distributions of $\Delta y^{+}$(nondimensional height of the first layer cell) for the two Reynolds numbers from the finest meshes. Because the maximum stretching ratio is not larger than 1.1, thus the present meshes satisfy the grid requirement for directly simulating the wall-bounded flow [41, 42]. 


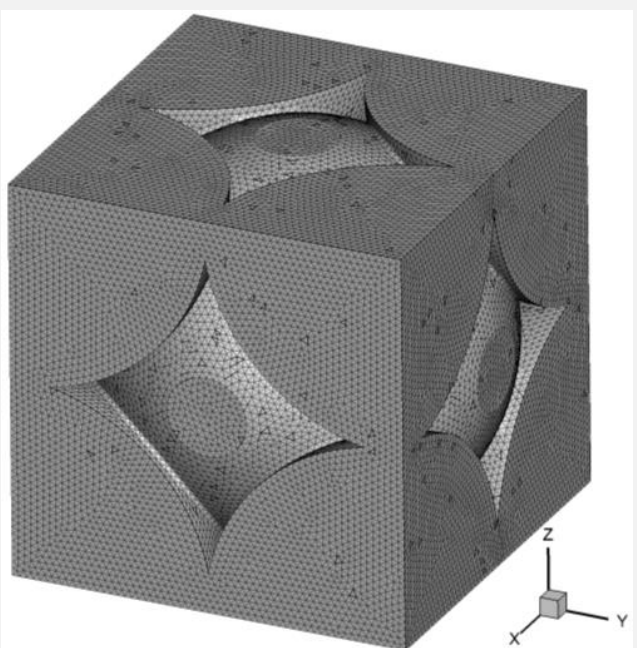

(a)

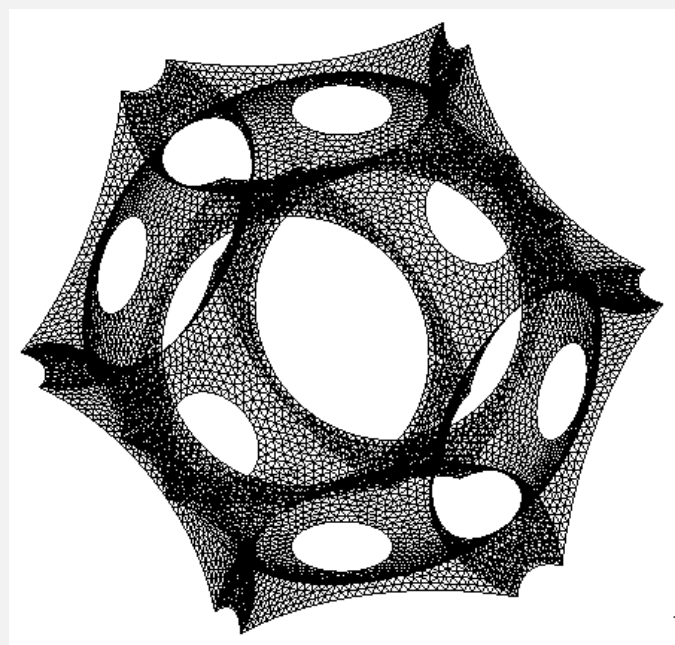

(b)

Fig. 2 Schematic of computational mesh: (a) computational domain and mesh; (b) solid structure

The time step $\Delta t$ decreases with the increase in the free stream velocity $U$, and we set $U \Delta t=2 \times 10^{-5} \mathrm{~m}$ in both simulations. Fig. 4 displays the distributions of the wall friction velocity, based on it we can obtain the non-dimensional time step $\Delta t^{+}=u_{\tau}^{2} \Delta t / v \sim \mathrm{O}(1)$.
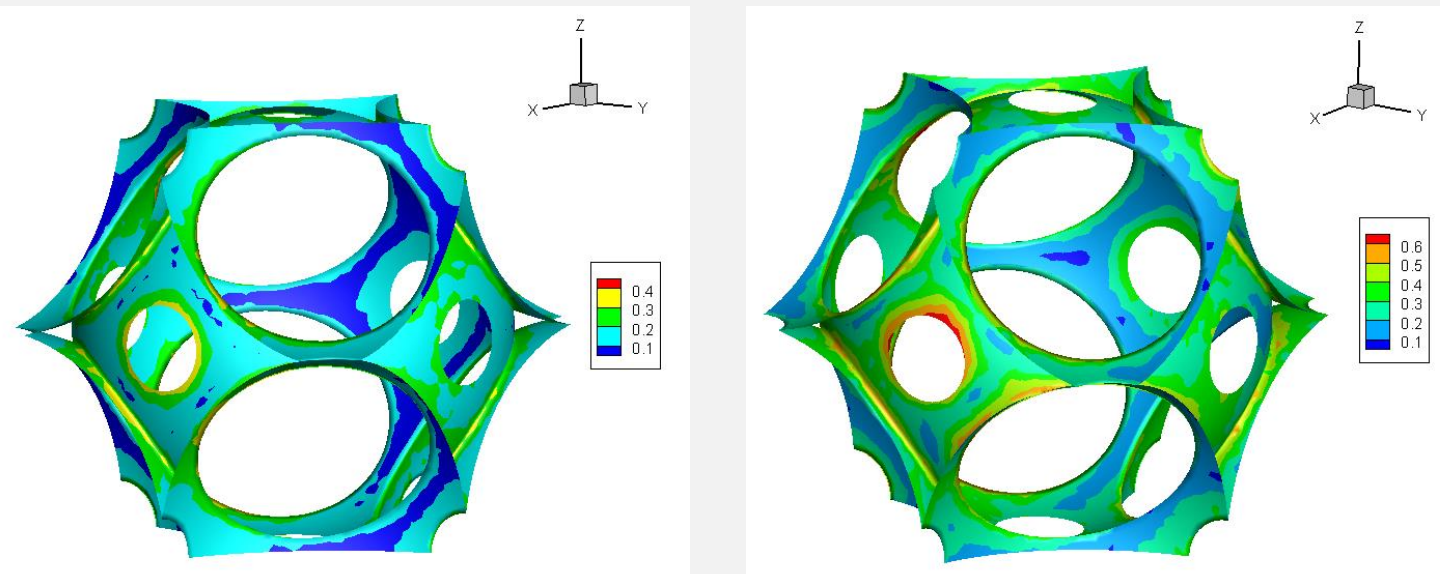

(a)

(b)

Fig. $3 \Delta y^{+}$distribution based on finest computational meshes: (a) $\operatorname{Re}=130$; (b) $\operatorname{Re}=250$ 

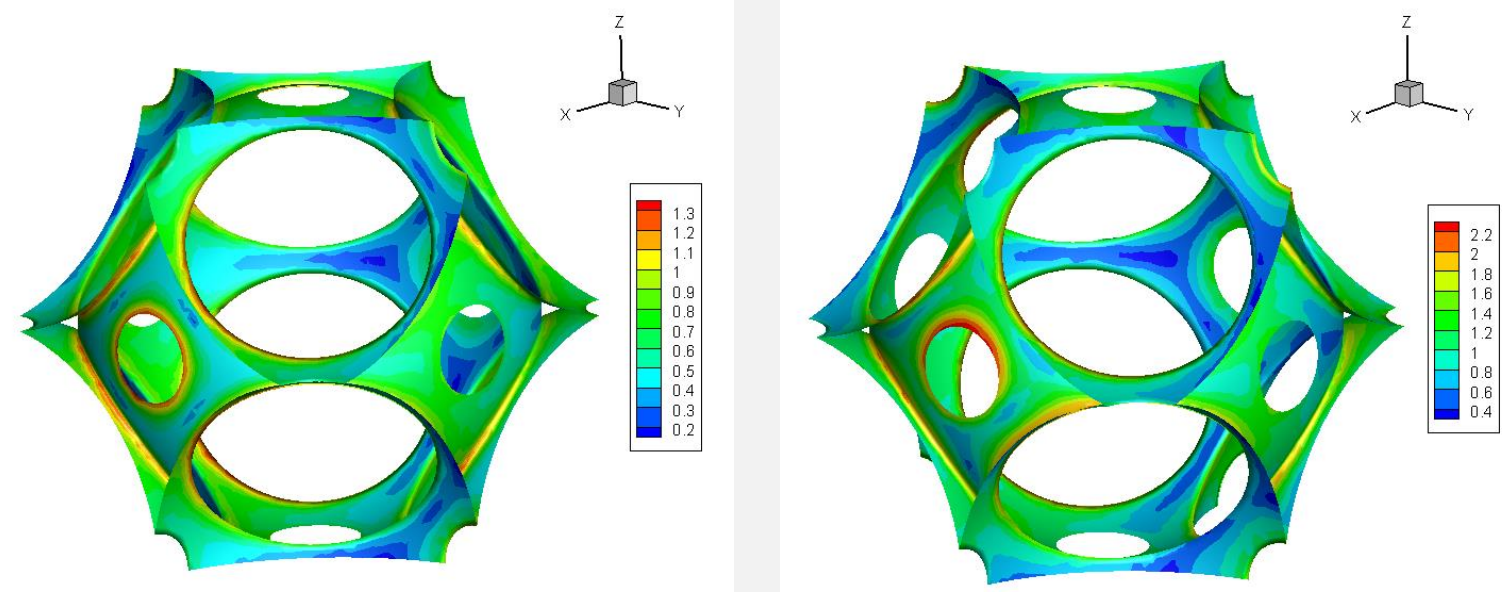

(a)

(b)

Fig. 4 Wall friction velocity (unit: m/s): (a) $R e=130$; (b) $R e=250$

\subsection{Numerical validation}

Once the flow has been fully developed, the permeability $K$ can be calculated by using the following equation:

$$
K=\frac{\mu U}{|\partial p / \partial x|}
$$

where $\mu$ is the dynamic viscosity, and $\partial p / \partial x$ is the pressure gradient in the streamwise direction. In the case of $\operatorname{Re}=130$, Fig. 5 presents the permeability calculated from numerical results based on the five sets of meshes as well as compared with the previous numerical [34] and experimental [43] results. The present numerical results show that the calculated permeability gradually approaches a constant with the increase in the cells of the computational meshes and the computational result nearly achieves a grid-independent solution at the finest meshes. Therefore, only results obtained by using the finest meshes are analyzed and discussed hereafter. 


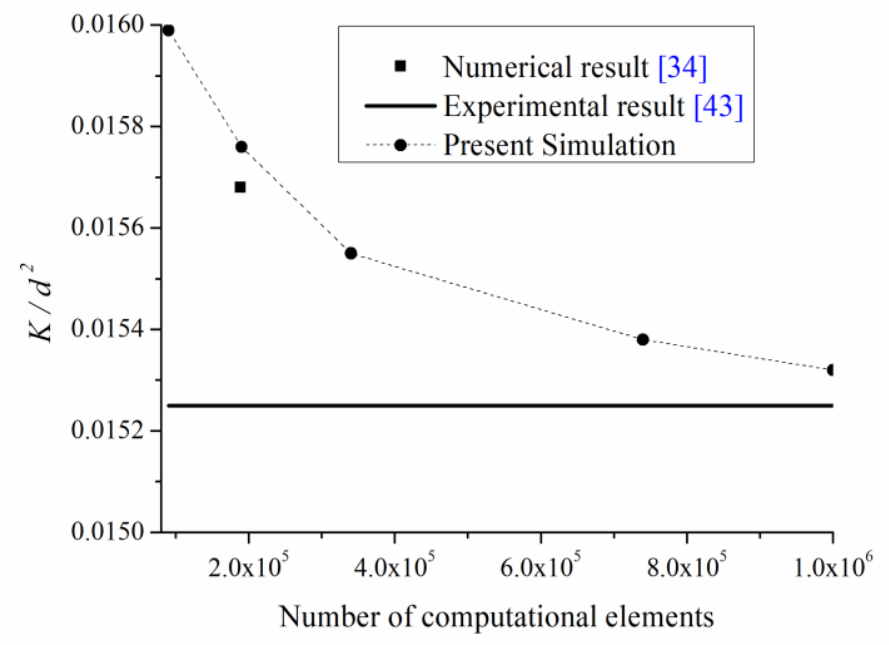

Fig. 5 Permeability calculated using different meshes and compared with existing experimental and numerical results

The Ergun's equation describes the relation among the pressure gradient, the geometrical parameters of the porous cell and flow velocity [27]. It has been widely used to estimate the pressure drop of the fluid through porous materials [44], such as granular media and foam materials, which is given by

$$
|\nabla p|=E_{1} \frac{\mu}{d_{p}^{2}} \frac{(1-\varepsilon)^{2}}{\varepsilon^{3}} U+E_{2} \frac{1-\varepsilon}{d_{p} \varepsilon^{3}} \rho_{0} U^{2}
$$

where $E_{1}$ and $E_{2}$ are Ergun constants, and are equal to 150 and 1.75 according to many experimental measurements [27]. $d_{p}$ is the mean particle diameter, which is different from the sphere diameter $D$ and the equivalent diameter $d$, and it depends on the types of porous materials, e.g. pellets, foams and honeycombs. For metal foams, the mean pore diameter $d_{p}$ is calculated as follows [45, 46]: 


$$
d_{p}=1.5 \times \frac{\frac{d}{2.3}\left[\frac{4}{3 \pi}(1-\varepsilon)\right]^{1 / 2}}{1-\left[\frac{4}{3 \pi}(1-\varepsilon)\right]^{1 / 2}}
$$

The two terms on the right hand side of Eq. (4) represent flow resistance from viscous and inertial contributions, and are named the viscous term and the inertial term, respectively. We can find that the viscous term dominates at low speed, while the inertial term becomes more prevalent with the increase in the flow velocity. Fig. 6 displays the variation of the pressure drop with Reynolds number, and a good agreement was achieved between the numerical results and the curve calculated from the Ergun's equation.

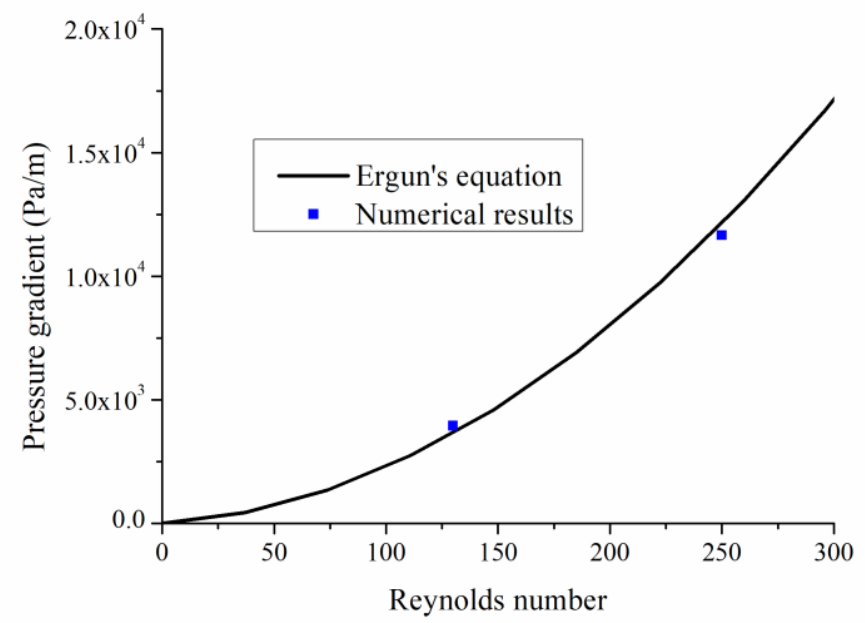

Fig. 6 Comparison of static pressure gradient between the numerical results and Ergun's equation

The above-mentioned comparison shows that the present numerical simulation with the finest mesh can accurately predict the permeability and pressure loss of the metal foam. A comparison of the unsteady flow features between the present simulation and some other studies, such as experimental measurements, would further give confidence of the present numerical study. However, as mentioned in Introduction, no relevant studies have been published due to the 
difficulties of experimental measurements. In this paper, we try to study numerically the unsteady flow features inside the cell of the metal foam because many previous numerical studies have confirmed that the flow simulation technique with an enough grid resolution can accurately capture the features of the wall-separated flow, such as flow through multi-perforated walls [47] and three-dimensional cavity flow with high Reynolds numbers [48].

\section{Results and analysis}

\subsection{Flow drag}

The total flow drag $L_{D}$ is defined by

$$
L_{D}=\int_{S} p \cos \theta d S+\int_{S} \tau \sin \theta d S
$$

where the first and second terms on the right hand side of Eq. (6) represent the pressure drag and friction drag, respectively. $\theta$ is the inclination angle between the normal direction of the solid surface and the free-stream velocity, $S$ is the area of the solid skeleton of the metal foam, $p$ and $\tau$ are the static pressure and shear stress acting on the solid surface, respectively.

Based on the above definition and flow simulation results, Fig. 7 and Fig. 8 plot time histories of the pressure and the friction drags at different Reynolds numbers. It is observed that Reynolds number has a great effect on the flow drag. Firstly, both the pressure and friction drags increase with the Reynolds number, as can be clearly seen from Eq. (4). Secondly, the ratio between the pressure drag and the friction drag increases with the Reynolds number, as shown in Table 1. This phenomenon can also be explained by Eq. (4), which indicates that the first term related to the fluid viscosity plays an important role on the pressure drop at a low flow velocity but the second term independent of the fluid viscosity has a major contribution to the flow drag at a high 
flow velocity. Thirdly, the Reynolds number affects the unsteadiness of the flow drag. The flow drag is nearly constant at $\operatorname{Re}=130$, but varies periodically at $\mathrm{Re}=250$.
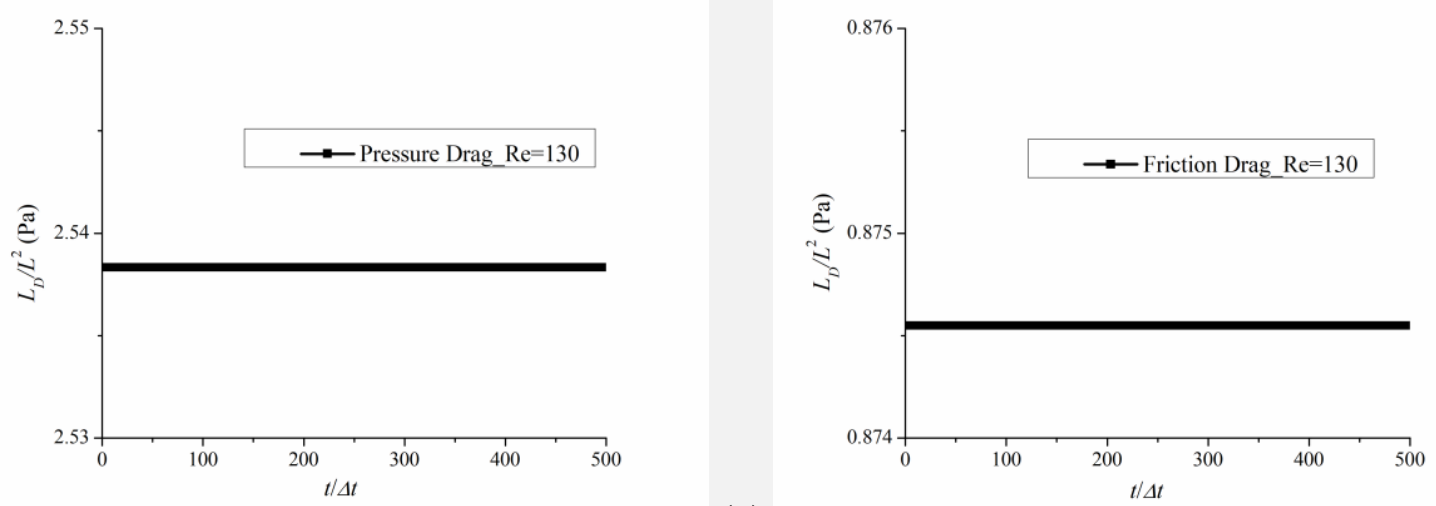

(a)

Fig. 7 Time histories of the flow drag at $\mathrm{Re}=130$ : (a) pressure drag; (b) friction drag
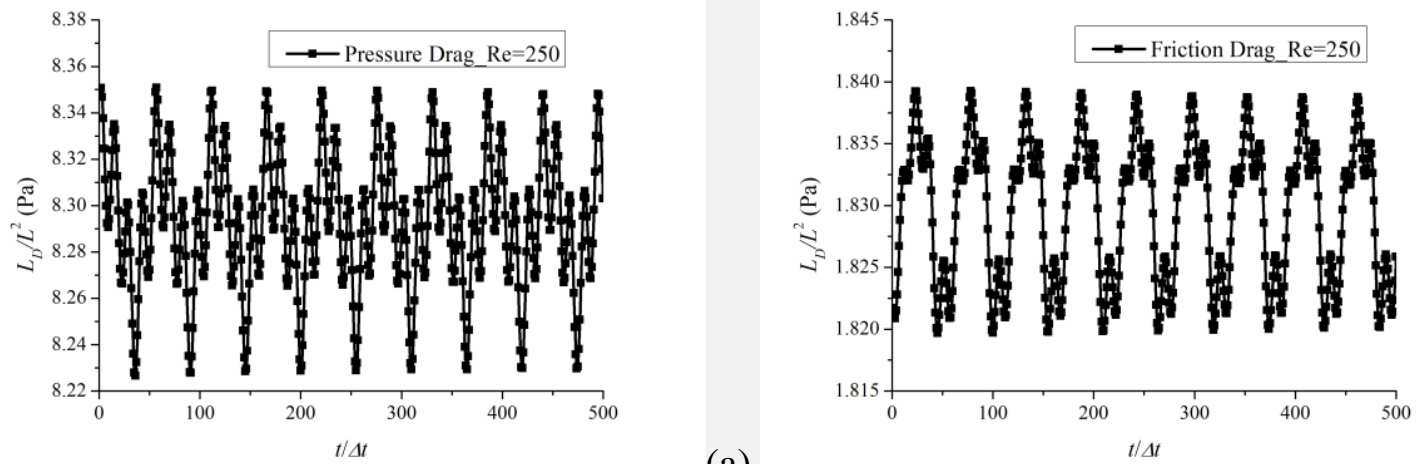

(a)

Fig. 8 Time histories of the flow drag at $\mathrm{Re}=250$ : (a) pressure drag; (b) friction drag

Table 1 Time-averaged pressure drag and friction drag

\begin{tabular}{cccc}
\hline Reynolds number & Pressure drag (N) & Friction Drag (N) & $\begin{array}{c}\text { Pressure-friction } \\
\text { Drag ratio }\end{array}$ \\
\hline 130 & $2.538 * 10^{-6}$ & $8.746 * 10^{-7}$ & 2.90 \\
250 & $8.289 * 10^{-6}$ & $1.823 * 10^{-6}$ & 4.55 \\
\hline
\end{tabular}


Fig. 8 indicates that fluctuations of both the pressure and friction drags are periodic at $\operatorname{Re}=250$. Because the pressure drag is highly dependent on the flow separation, the above feature implies that periodic flow separation occurs at $\operatorname{Re}=250$ but only steady flow separation occurs at $\operatorname{Re}=130$. Detailed analysis on the flow separation will be given later. Fig. 9 shows the spectra of the pressure and friction drags at $\mathrm{Re}=250$. Both the pressure and friction drags display discrete peaks at a frequency of $3040 \mathrm{~Hz}$ and its higher harmonics. This feature implies that the flow through the OCMF would generate tonal noise, which negatively affects the noise reduction performance of the OCMF.

The above results confirm that the flow drag of the OCMF is predominantly caused by the pressure drag rather than the friction drag. Therefore, parameters related to the friction drag, such as the surface area-volume ratio and the fluid viscosity, are insufficient to describe the total flow drag. Moreover, the pressure drag is usually highly related to the flow separation, thus it is necessary to investigate the detailed pore-scale flow in order to reveal the mechanism of flow loss.

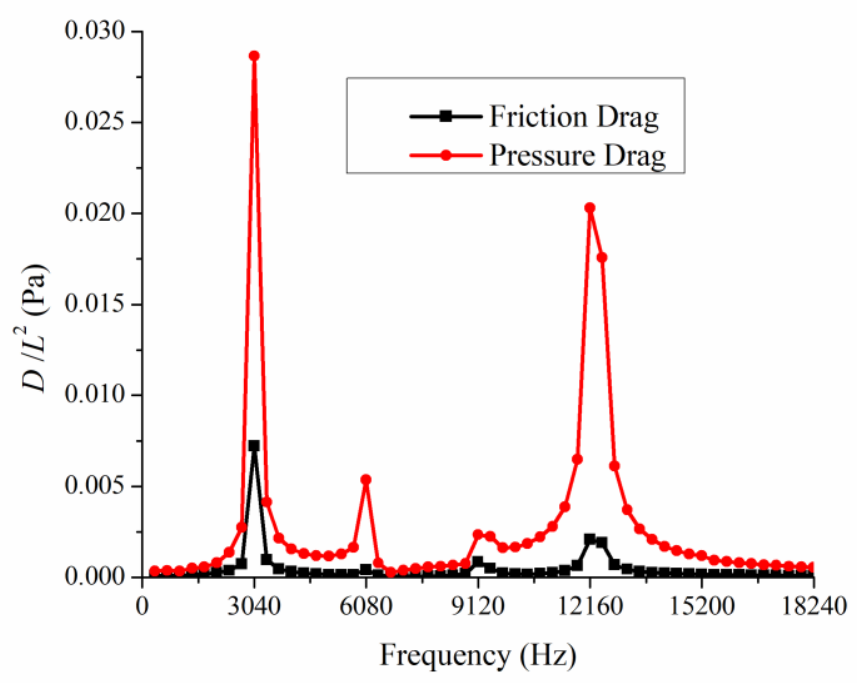

Fig. 9 Spectra of the pressure drag and friction drag at $R e=250$ 


\subsection{Pore-scale flow}

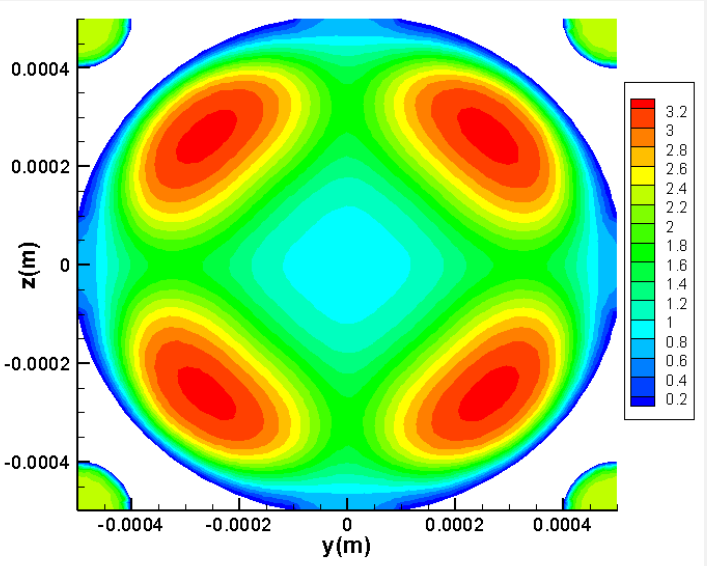

(a)

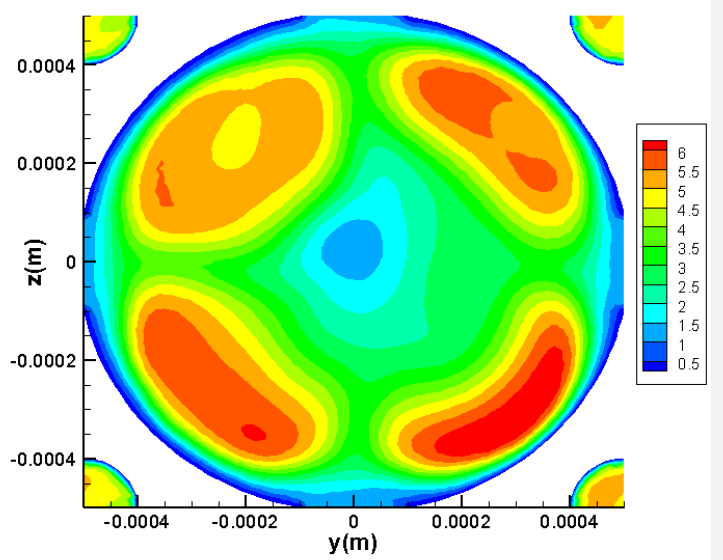

(b)

Fig. 10 Contours of instantaneous velocity magnitude at surface $x=0$ (unit: $\mathrm{m} / \mathrm{s}$ ): (a) $\operatorname{Re}=130$; (b) $\operatorname{Re}=250$.

Fig. 1(b) illustrates four representative cross-sections inside the computational domain, and flow field in these cross-sections is analyzed below. Fig. 10 displays contours of the instantaneous velocity magnitude on the surface of $x=0$, which is perpendicular to the free-stream velocity. At $\operatorname{Re}=130$, the flow velocity is symmetric to both surfaces of $y=0$ and $z=0$, but the flow velocity becomes asymmetric at $\mathrm{Re}=250$. The instantaneous flow pattern indicates that the flow is steady at $\mathrm{Re}=130$, which is confirmed by the time histories of flow quantifies but the flow is unsteady at $\operatorname{Re}=250$ which leads to the asymmetric instantaneous flow. 

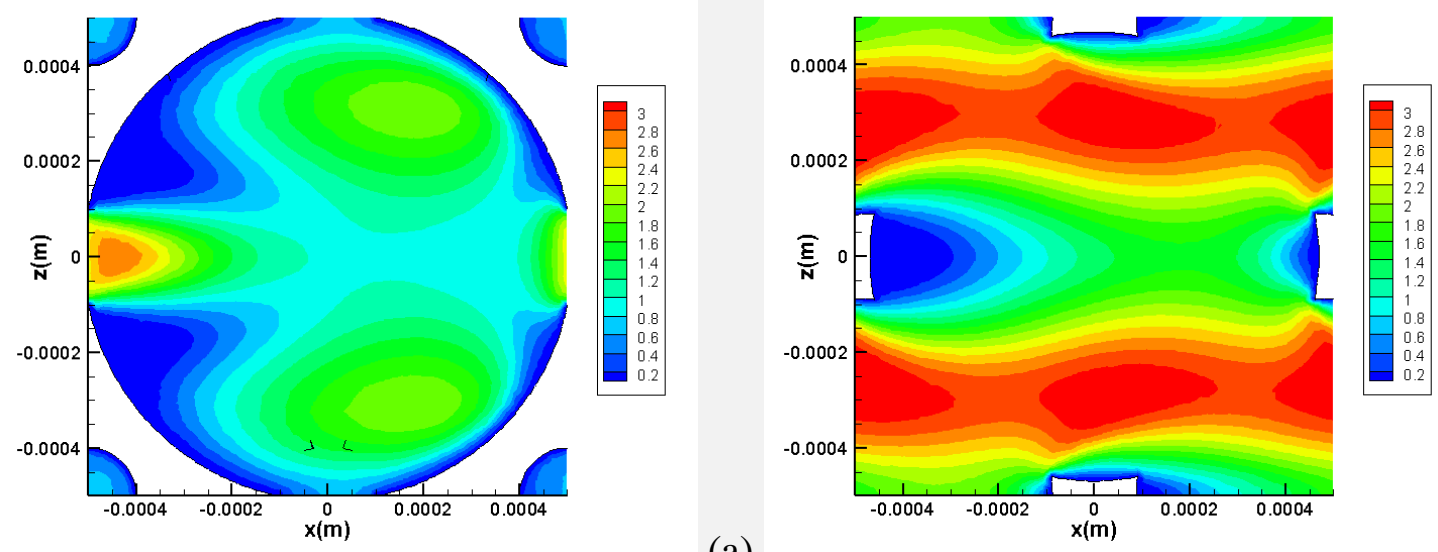

(a)

(b)

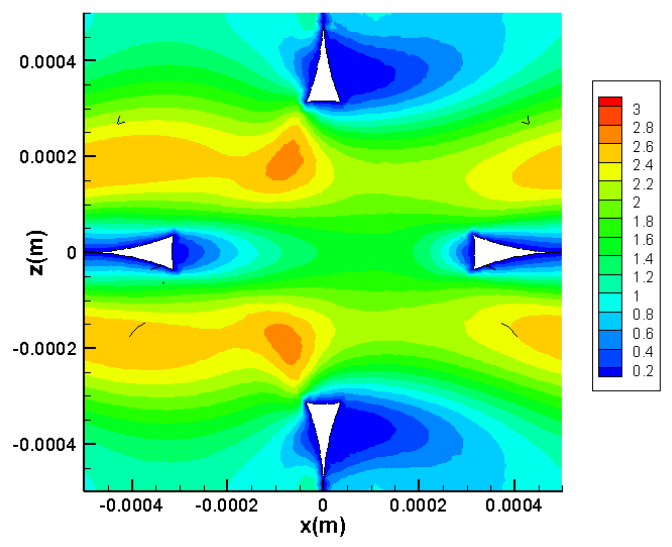

(c)

Fig. 11 Contours of instantaneous velocity magnitude at $\operatorname{Re}=130$ (unit: $\mathrm{m} / \mathrm{s}$ ): (a) $y=0$; (b) $y=0.2 \mathrm{~mm}$ and (c) $y=0.4 \mathrm{~mm}$

As shown in Fig. 11, the instantaneous velocity fields on the three surfaces parallel to the freestream are displayed for $\mathrm{Re}=130$. The flow at this Reynolds number is steady, thus the instantaneous flow field is the same as the time-averaged flow field. The steady vortices are the most significant flow feature in this situation, and this phenomenon is similar to the laminar flow separation of the cylinder flow at the Reynolds number in the range from 15 to 40 . At surface $y=0$, a symmetric jet flow whose centerline is $\mathrm{z}=0$ causes the two symmetric separation regions owing to the shear effect of velocity gradient. At surface $y=0.2 \mathrm{~mm}$, symmetric shedding vortex 
regions are formed behind the foam structure. At surface $y=0.4 \mathrm{~mm}$, although flow separations occur behind the two wedges, the flow is still symmetric. This phenomenon induces a steady pressure drag acting on the solid surfaces, as shown in Fig. 7. That is to say, the steady flow separation only increases the total flow drag but does not generate aerodynamic noise.

However, the flow field becomes highly unsteady at $\mathrm{Re}=250$, as shown in Fig. 12-Fig. 14, where $T$ is the time of flow through a single cell which is calculated by $T=L / U$. At surface $y=0$, the fluid through the structure forms a jet flow, which is not symmetric any more. The periodic swing of the jet is accompanied with successive flow separations at the two sides of the jet shear layer. Meanwhile, the periodic alternative vortex shedding from the structure surfaces is a significant flow feature at surfaces $y=0.2 \mathrm{~mm}(L / 5)$ and $y=0.4 \mathrm{~mm}(2 L / 5)$. As we known, Strouhal number, which is the frequency of the vortex shedding non-dimensionalized by the flow velocity and the characteristic length of the bluff body, is usually used to describe the unsteadiness of the alternative vortex shedding. Usually, the length of the obstacle projected to the plane normal to the flow direction is used as the characteristic length. We can find that the characteristic lengths of the skeleton on these three surfaces are different because the skeletons are of significantly different cross-section shapes. However, seen from Fig. 12-Fig. 14, the periods of the velocity fluctuation in these surfaces are the same. In order to quantitatively analyze the vortex shedding feature, time histories of flow velocity at three typical monitors are plotted in Fig. 15, and the corresponding spectra of the flow velocity are given in Fig. 16. The results confirm that the components of the flow velocity at those three different positions have the same period of the vortex shedding, and the corresponding vortex shedding frequency is $3040 \mathrm{~Hz}$. 




(a)
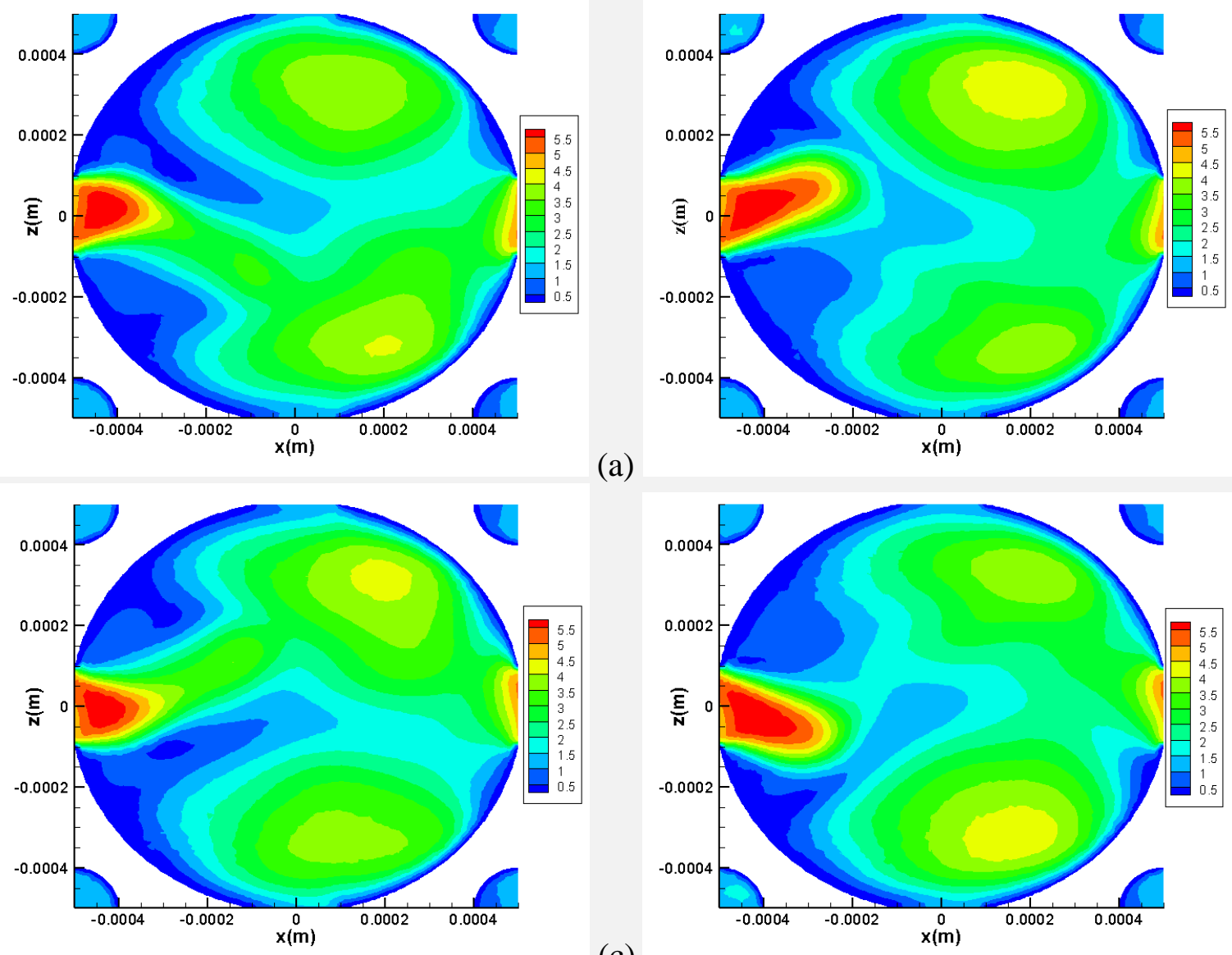

(c)

(b)

(d)

Fig. 12 Contours of instantaneous velocity magnitude at $\operatorname{Re}=250$ and $y=0$ (unit: $\mathrm{m} / \mathrm{s}$ ): (a) $T / 4$; (b) $T / 2$; (c) $3 T / 4$; (d) $T$
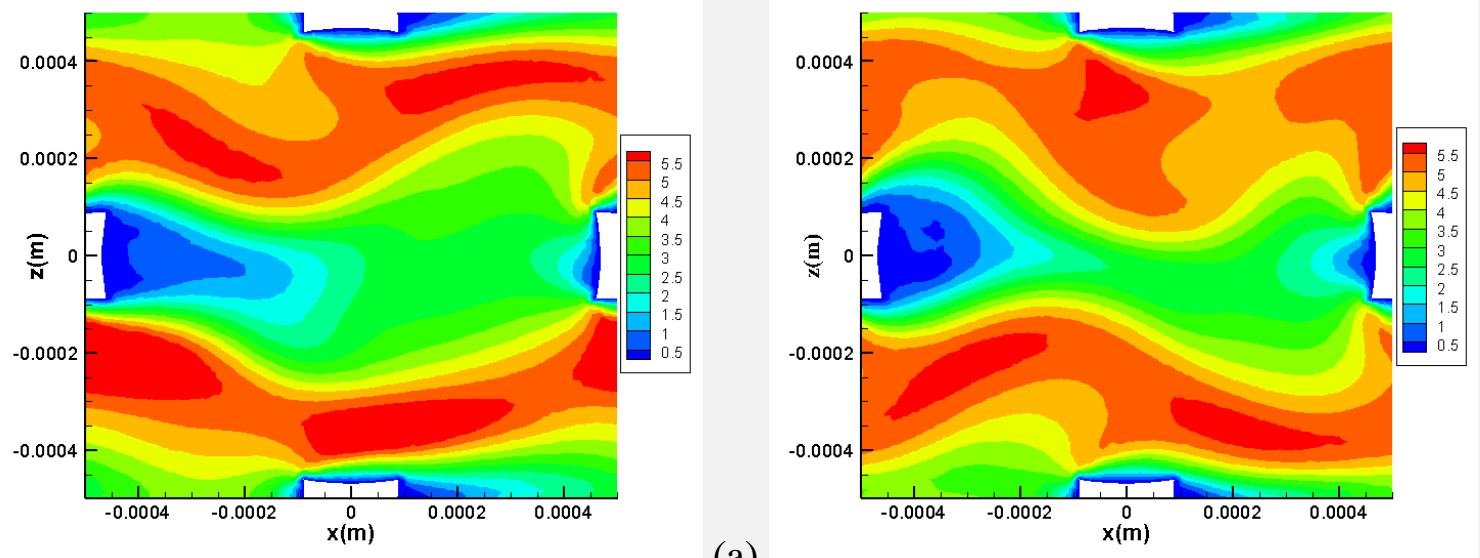

(a)

(b) 

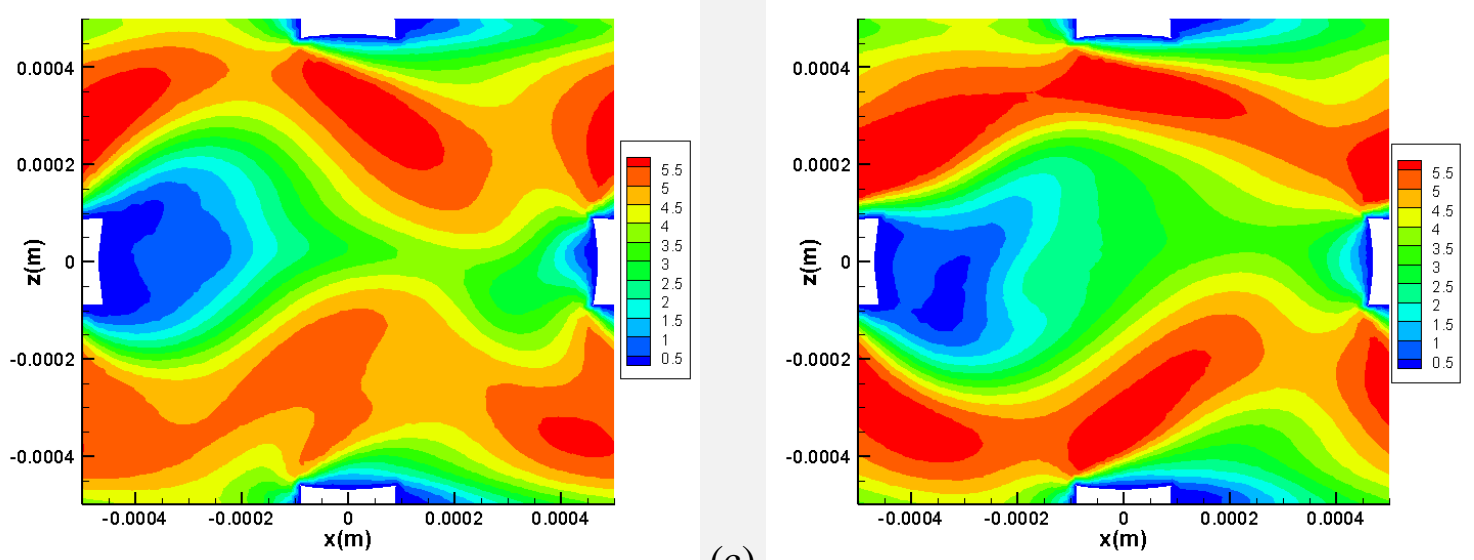

(c)

Fig. 13 Contours of instantaneous velocity magnitude at $\operatorname{Re}=250$ and $y=0.2 \mathrm{~mm}$ (unit: $\mathrm{m} / \mathrm{s}$ ): (a)

$T / 4$; (b) $T / 2$; (c) $3 T / 4$; (d) $T$

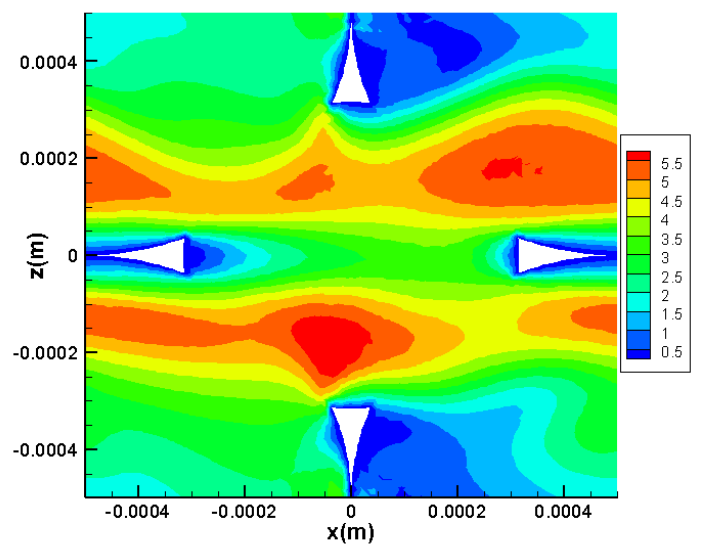

(a)

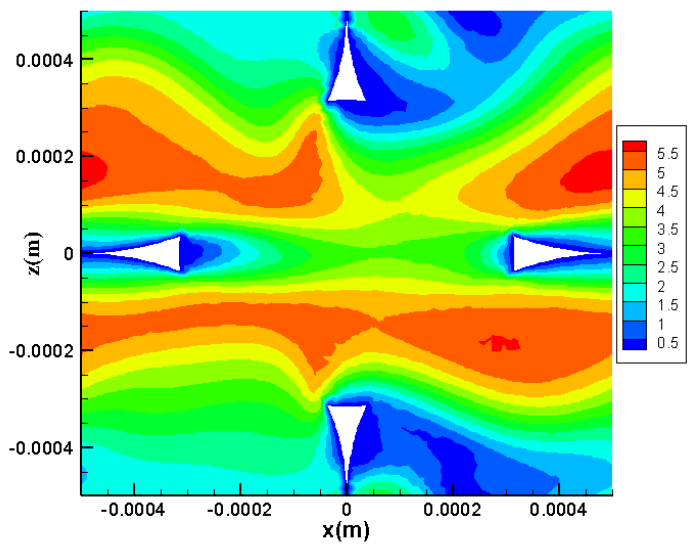

(b)
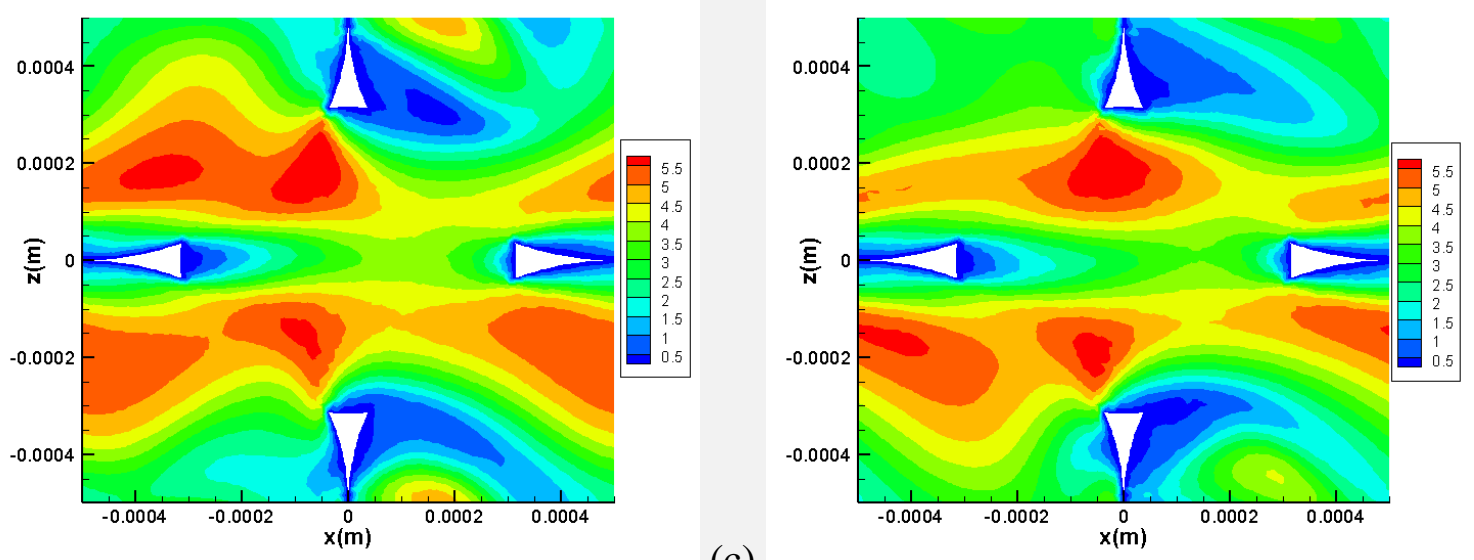

(c)

(d)

Fig. 14 Contours of instantaneous velocity magnitude at $\mathrm{Re}=250$ and $y=0.4 \mathrm{~mm}$ (unit: $\mathrm{m} / \mathrm{s}$ ): (a)

$T / 4$; (b) $T / 2$; (c) $3 T / 4$; (d) $T$ 

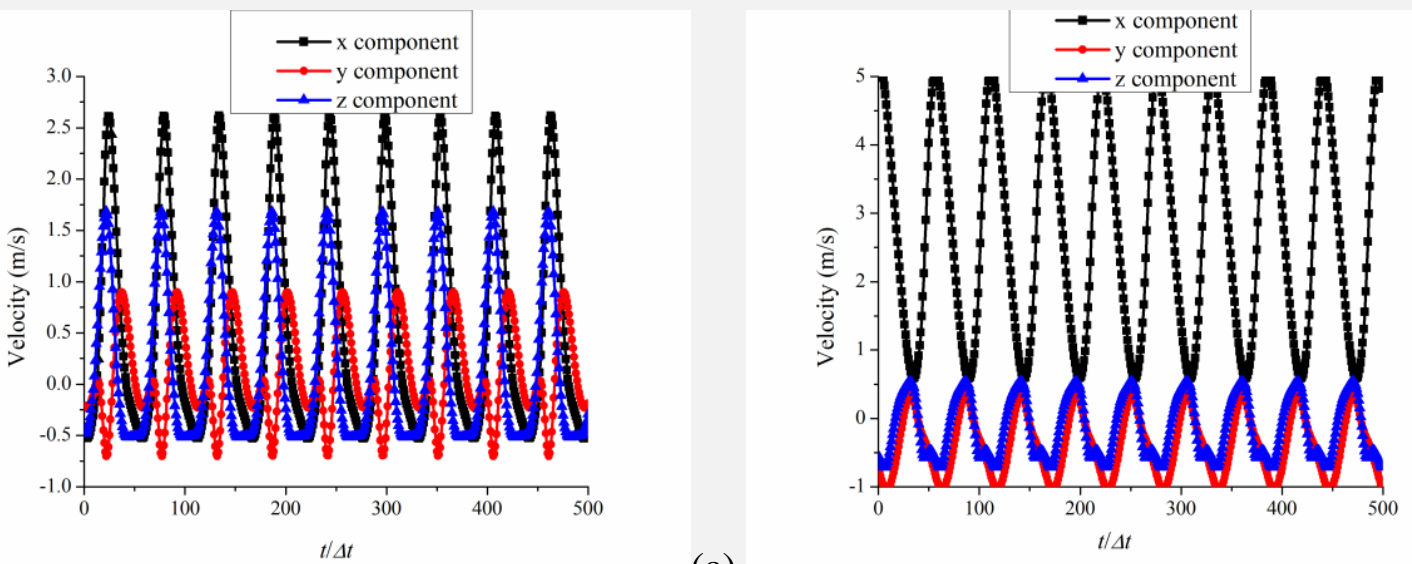

(a)

(b)

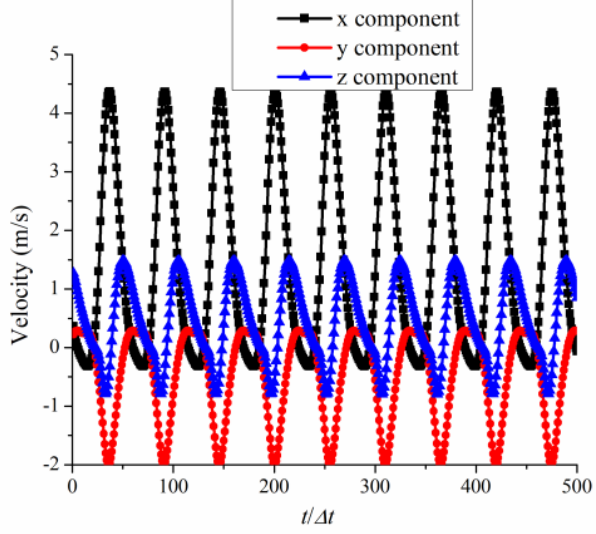

(c)

Fig. 15 Time histories of the flow velocity components at three characteristic points:

(a) $(-0.3 \mathrm{~mm}, 0,0.15 \mathrm{~mm})$; (b) $(-0.3 \mathrm{~mm}, 0.2 \mathrm{~mm}, 0.15 \mathrm{~mm})$; (c) $(0.2 \mathrm{~mm}, 0.4 \mathrm{~mm}, 0.5 \mathrm{~mm})$
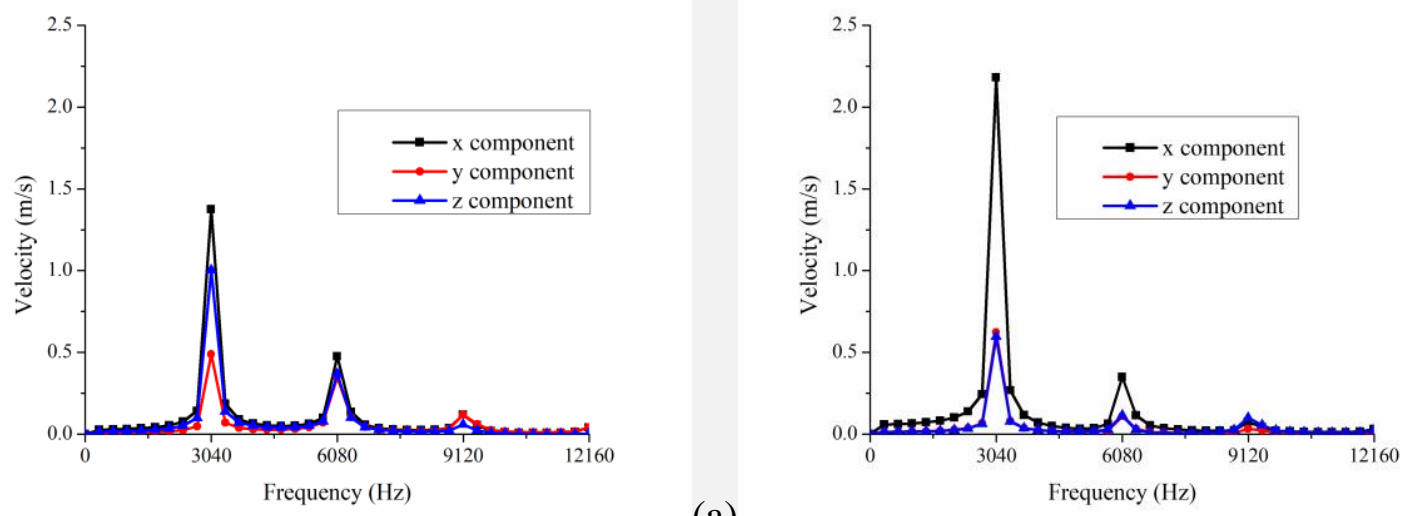

(a) 


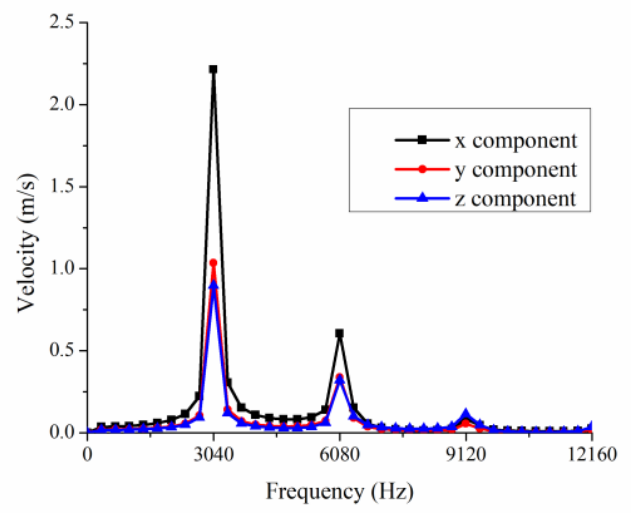

(c)

Fig. 16 Spectra of the flow velocity components at three characteristic points:
(a) $(-0.3 \mathrm{~mm}, 0,0.15 \mathrm{~mm}) ;($ b) $(-0.3 \mathrm{~mm}, 0.2 \mathrm{~mm}, 0.15 \mathrm{~mm}) ;(\mathrm{c})(0.2 \mathrm{~mm}, 0.4 \mathrm{~mm}, 0.5 \mathrm{~mm})$

Fig. 17 displays iso-surfaces of the instantaneous streamwise velocity at $u_{x}=0$, and the flow separation regions (green shade areas) are clearly founded in both cases. The first separation region is located near the inlet cross section $x=-0.5 \mathrm{~mm}$. High-speed jet flows from the central circular hole causes the flow separation behind the skeleton. The second separation region appears near the cross-section $x=0$, and the flow passing the bluff skeleton forms four separation sub-regions, which are symmetric at $\mathrm{Re}=130$ but becomes asymmetric at $\mathrm{Re}=250$, and this feature is consistent with the aforementioned flow feature shown in velocity contours.

Moreover, Fig. 18 shows contours of the instantaneous wall shear stress in the $x$ direction. The regions of the negative shear stress coincide with the flow separation regions that are shown in Fig. 17. Although negative shear stress due to the flow separation reduces the friction drag, the pressure drag is predominant for the OCMF studied in this paper, thus the reduction of the friction drag has little effect on the total drag. 


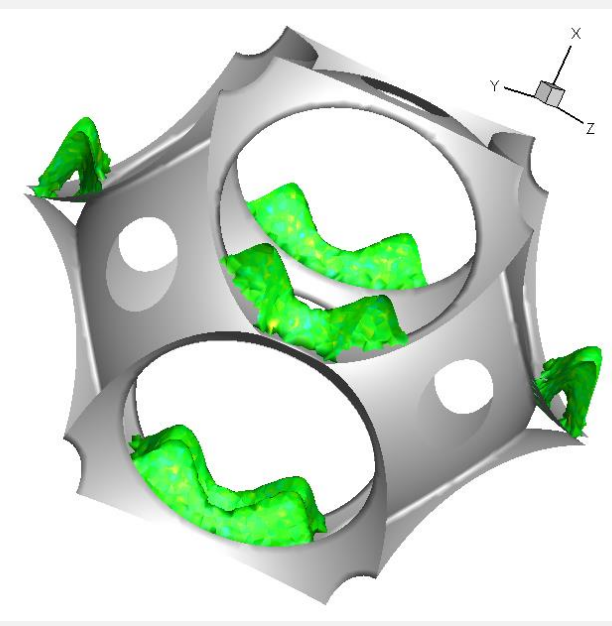

(a)

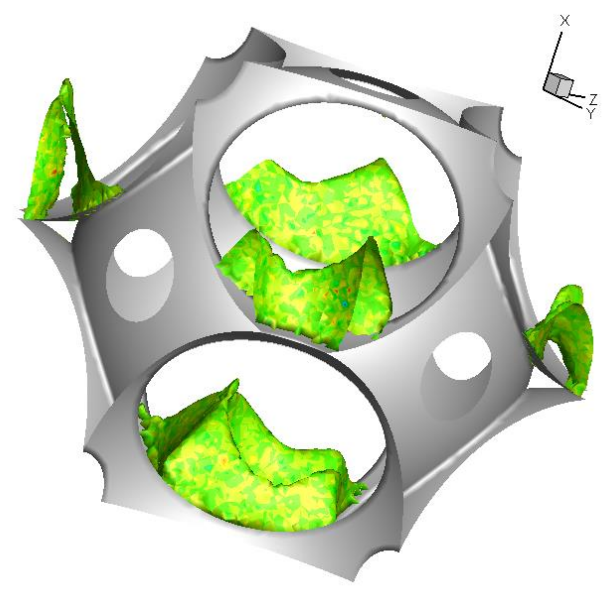

(b)

Fig. 17 Snapshot of iso-surfaces of $u_{x}=0$ : (a) $\mathrm{Re}=130$; (b) $\mathrm{Re}=250$
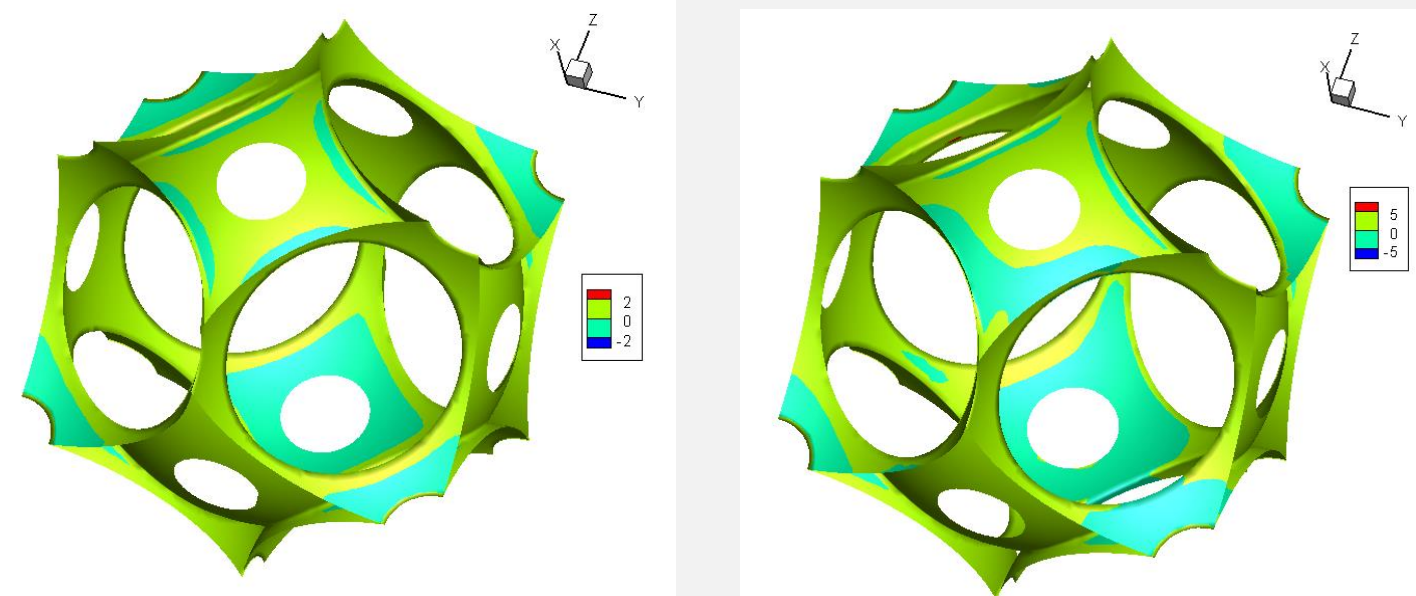

(a)

(b)

Fig. 18 Contour of instantaneous wall shear stress in $x$ direction: (a) $\operatorname{Re}=130$; (b) $\operatorname{Re}=250$

\subsection{Aerodynamic noise}

The acoustic analogy proposed by Lighthill [49] and further developed by Curle [50] is used to analyze aerodynamic noise radiated from flow and its interaction with the cell structure of the OCMF. As the flow Mach number within the porous material is very small, contributions to far field noise from quadrupole sources are much smaller than from dipole sources. Only the noise radiated from the pressure fluctuations acting on the stationary solid surfaces is calculated, thus 
the Curle's equation [50] is used to predict the broadband noise spectrum and the directivity pattern. 36 observers are uniformly located at a circle normal to the flow direction, with the center at the coordinate origin and with a diameter of $1 \mathrm{~m}$. Since the characteristic length of the single cell $L$ is much less than $|\mathbf{x}|$, where $\mathbf{x}$ is the position vector of the observers, we can use the following simplified equation to compute the time-domain sound pressure $p_{a}^{\prime}$ :

$$
4 \pi p_{a}^{\prime}(\mathbf{x}, t)=\frac{\partial}{\partial t} \int_{\Gamma} \frac{r_{i} n_{i} p(\mathbf{y}, \tau)}{c_{0} r^{2}} d \Gamma(\mathbf{y})+\int_{\Gamma} \frac{r_{i} n_{i} p(\mathbf{y}, \tau)}{r^{3}} d \Gamma(\mathbf{y})
$$

where $\Gamma$ denotes the skeleton surface of the metal foam, $r_{i}=x_{i}-y_{i}$ is the $i$ th component of source-observer radiation vector, and $n_{i}$ is the $i$ th component of the unit normal vector on the skeleton surface $\Gamma$.

Fig. 19 displays the sound pressure spectra of the observer at the downstream position of $(1 \mathrm{~m}, 0,0)$. Note that sound radiated from only one single cell is calculated in this study, thus the amplitude of the sound pressure is very low. Here, we focus on the study of the noise spectrum features. It can be observed from Fig. 19 that discrete peaks appear at the frequency of $3040 \mathrm{~Hz}$ and its higher harmonics, this feature is consistent with that of the pressure drag shown in Fig. 9. However, the acoustic pressure amplitude at $12160 \mathrm{~Hz}$ is higher than that at $3040 \mathrm{~Hz}$, this is owing to the more significant contribution of the pressure fluctuation at high frequencies than that at low frequencies, as revealed by the following frequency-domain equation obtained from the Fourier transformation over the time-domain integral Eq. (7):

$$
4 \pi p_{a}^{\prime}(\mathbf{x}, \omega)=\int_{\Gamma}\left(i \omega r / c_{0}+1\right) \frac{r_{i} n_{i} p(\mathbf{y}, \omega) e^{j \omega r / c_{0}}}{r^{3}} d \Gamma(\mathbf{y})
$$


Eq. (8) indicates that sound pressure received by a specific observer $x$ depends on both the frequency-domain source strength $p(\mathbf{y}, \omega)$ and the angular frequency $\omega$. Although, an shown in Fig. 9, the component of the frequency-domain pressure drag at $3040 \mathrm{~Hz}$ is about 1.5 times of that at $12160 \mathrm{~Hz}$, Fig. 19 indicates that the acoustic pressure amplitude at $12160 \mathrm{~Hz}$ is much higher than that at $3040 \mathrm{~Hz}$ due to the various contributions of the frequency factor $i \omega r / c_{0}+1$ in Eq. (8)

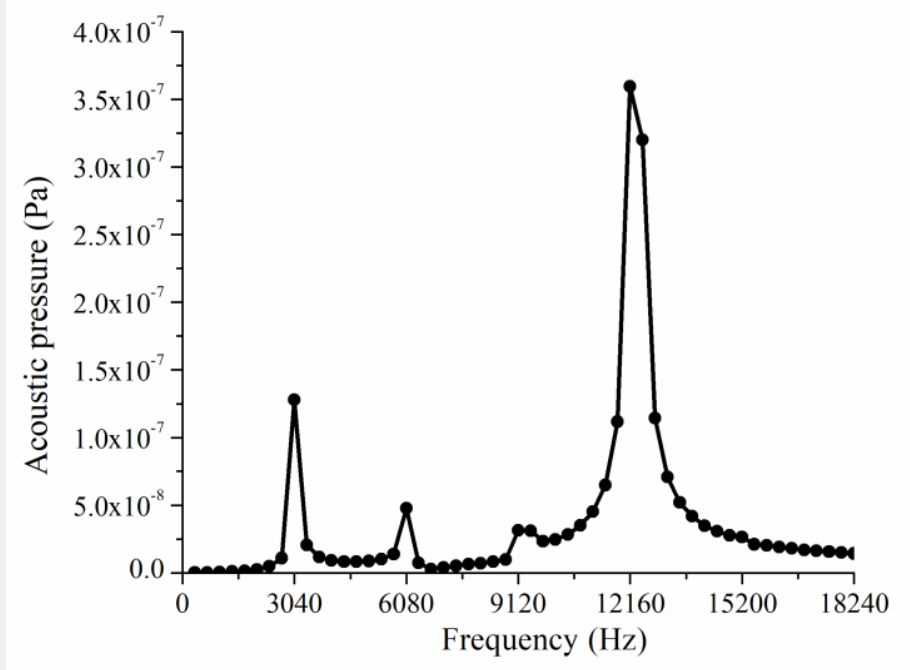

Fig. 19 Spectrum of sound pressure radiated from the dipole source on skeleton surface

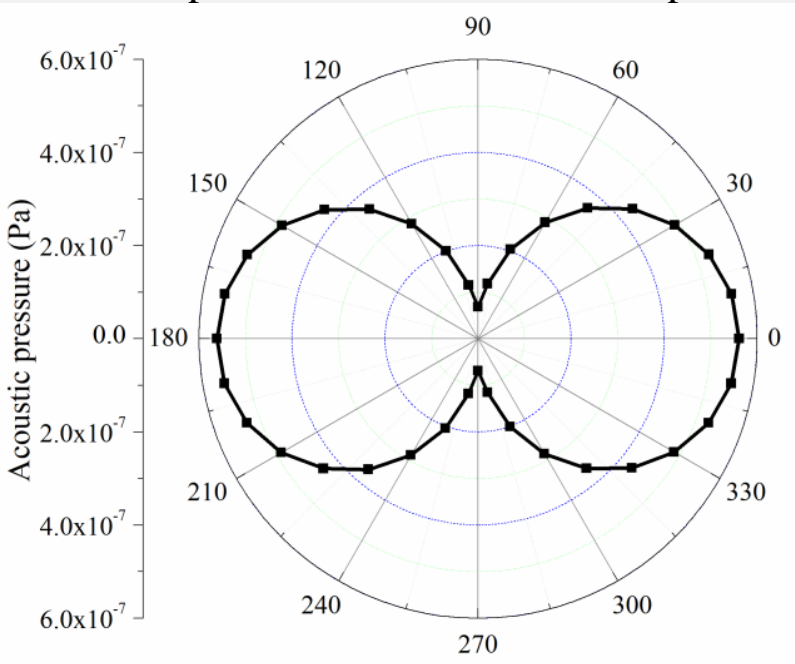

Fig. 20 Directivity pattern of the sound pressure 
Fig. 20 displays the directivity pattern of the overall sound pressure, where 0 and $180^{\circ}$ correspond to the downstream and upstream directions, respectively. As mentioned above, a single cell of the metal foam can be regarded as an acoustically compact dipole source owing to its length usually much less than the acoustic wavelength. Therefore, the acoustic radiation feature of a single cell satisfies the classic " $\infty$ " feature of a point dipole source, as shown in Fig. 20. The dipole axis is parallel to the flow direction, implying the noise is mainly contributed from the fluctuation of the flow drag. This directivity pattern is different to that of noise generated by the unsteady flow separation from a circular cylinder, and the maximum noise radiation direction in the latter case is perpendicular to the flow direction due to the strong fluctuations of the lift acting on the cylinder surface.

The root mean square of the wall pressure fluctuations is illustrated in Fig. 21. It can be seen that intense wall pressure fluctuations appear near the flow separation regions shown in Fig. 17, thus it further confirms that the wall pressure fluctuations, i.e., acoustic dipole sources, are mainly stimulated by the flow separations inside the cell. Moreover, as shown in Fig. 21, the wall normal vector for the high wall pressure fluctuation region is nearly parallel to the flow direction, therefore it is expected that the maximum noise radiation direction is parallel to flow direction according to Eq. (8), and this feature is consistent with the numerical result shown in Fig. 20.

The wall pressure fluctuations at two different frequencies $3040 \mathrm{~Hz}$ and $6080 \mathrm{~Hz}$ are illustrated in Fig. 22. It is found that the intense wall pressure fluctuations are located near the flow separation regions at both frequencies, implying that tonal noise components at the vortex shedding frequency and its first harmonics are mainly caused by the fluctuations of the pressure drag. It should be noted that this feature is also different from that of the unsteady flow separation from a circular cylinder. As is well known, for unsteady flow past a circular cylinder, 
the periodic flow separation causes intense lift fluctuations at the vortex shedding frequency and the intense drag fluctuations at the double vortex shedding frequency, thus the maximum noise direction is perpendicular to the flow direction at the vortex shedding frequency but is parallel to the flow direction at the double vortex shedding frequency. For the numerical case studied in this paper, the unsteady flow separation only stimulates intense drag fluctuations at both the vortex shedding frequency and its first harmonics, thus the maximum noise radiation direction is always parallel to the flow direction.

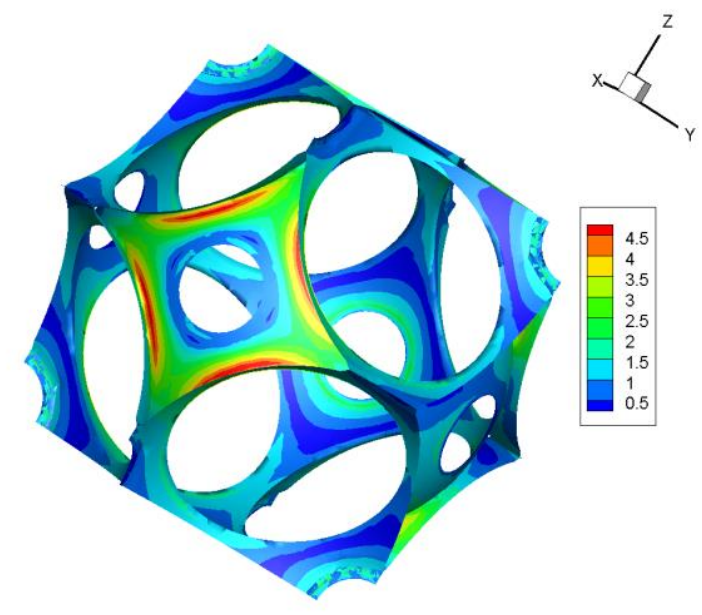

Fig. 21 Root mean square of the wall pressure fluctuations
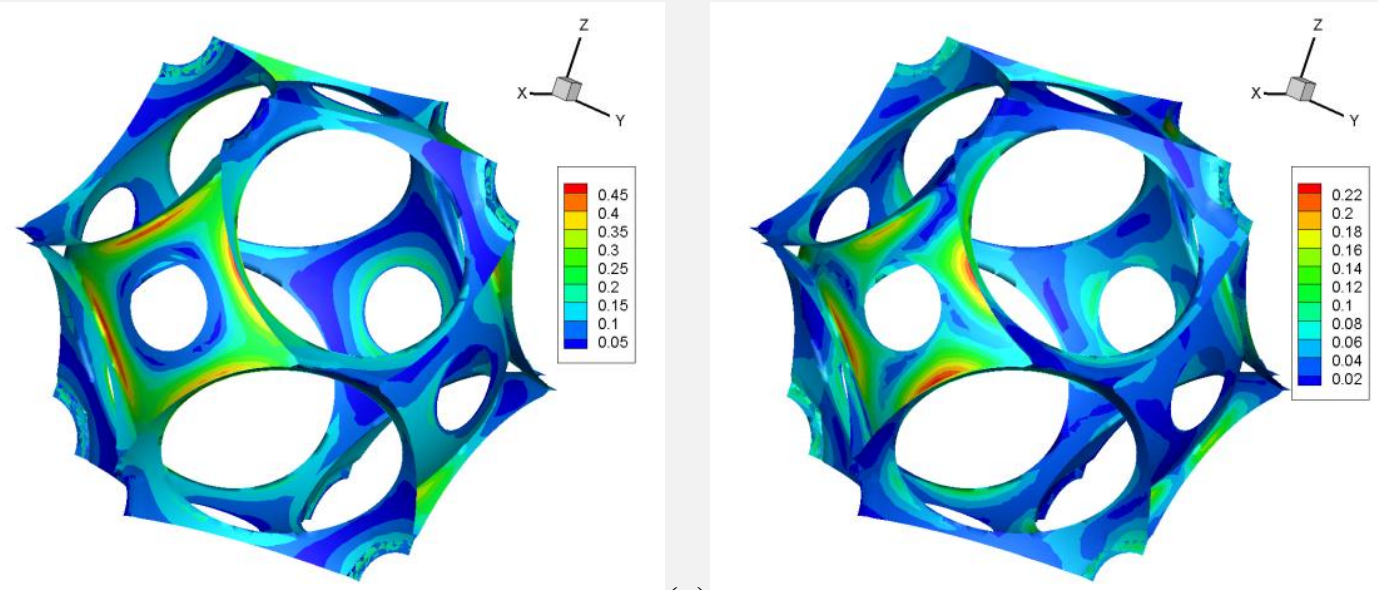

(a)

Fig. 22 Components of the wall pressure fluctuations: (a) $3040 \mathrm{~Hz}$; (b) $6080 \mathrm{~Hz}$ 


\section{Conclusion}

Low-Reynolds-number pore-scale flow through a single cell of the metal foam was studied numerically, and the Curle's acoustic analogy was used to analyze the related aeroacoustics features. Compared with the existing experimental and numerical investigations, the present research attempts to reveal the detailed pore-scale flow physics inside the cell, thus some new flow patterns are found and the corresponding physical mechanism are clarified. Conclusions are drawn as follows.

(1) Numerical results of the pore-scale flow inside the metal foam reveal that the pressure drag is usually much higher than the friction drag despite a very high surface area-volume ratio of the metal foam. The pressure drag is mainly related to two flow separation regions for the studied metal foam. The first is located behind the skeleton within a small circular pole, from where a jet flow causes the flow separation. The second region is behind the cross-section which has the maximum through-flow area, and the flow separation can be observed behind the bluff skeleton. Presence of flow separation regions has a negative effect on the application of OCMF, because it greatly increases the pressure drag while only slightly reduces the friction drag.

(2) The Reynolds number has a great impact on the flow separation in the cell of the metal foam. At $\operatorname{Re}=130$, the flow separation is steady, and fixed pairs of vortices occur inside the cell of the metal foam. This type of separation bubble only forms the steady pressure drag, thus has no negative effect on reducing the aerodynamic noise. At $\operatorname{Re}=250$, periodic alternating shedding vortices are visible. This phenomenon causes that the pressure drag is unsteady, and the periodic alternating shedding vortices form the pressure fluctuation on the skeleton of the metal foam and finally induces additional tonal noise. 
(3) The alternative vortices shedding from different surfaces have the same frequency, and this vortex shedding frequency is the same as the pulsating frequency of the flow drag, thus revealing the mechanism of the vortex shedding and suppressing this flow pattern are beneficial to reduce the flow drag of the metal foam as well as to further improve the noise-reduction performance. Although the pressure drag is predominant at the vortex shedding frequency, the tonal noise is predominant at a high harmonics of the vortex shedding frequency. This phenomenon is owing to sound contributed from the pressure fluctuation at high frequencies larger than that at low frequencies.

(4) The unsteady flow and aeroacoustics features are greatly different from those of the cylinder flow. The unsteady flow separation from the cylinder stimulates fluctuations of both the lift and drag, but the flow separation inside the cell mainly stimulates strong fluctuations of the pressure drag. For noise generated from a cylinder flow, tonal components at the vortex shedding frequency and its first harmonics are caused by the lift and drag fluctuations, respectively. This feature causes that the maximum noise radiation direction at these two frequencies are perpendicular and parallel to the flow direction, respectively. However, the unsteady flow separation inside the porous cell mainly stimulates drag fluctuations which are the dominant aeroacoustic sources, thus the maximum noise radiation direction is always parallel to the flow direction.

\section{Acknowledgments}

The authors gratefully acknowledge high-performance computing facilities IRIDIS4 at the University of Southampton in the completion of this work. The research is supported by the National Natural Science Foundation of China (No. 51806164, 51511130075), International Postdoctoral Exchange Fellowship Program (No. 20160101), Natural Science Basic Research 
Plan in Shaanxi Province of China (No. 2018JQ5185) and Newton Fund (No. IE141516). The valuable comments from the editor-in-chief, Prof. John Ekaterinaris, and anonymous reviewers are grateful for improving the quality of the paper.

\section{References}

[1] R. Dyga, M. Płaczek, Heat transfer through metal foam-fluid system, Experimental Thermal and Fluid Science, 65 (2015) 1-12.

[2] K. Boomsma, D. Poulikakos, F. Zwick, Metal foams as compact high performance heat exchangers, Mechanics of materials, 35 (2003) 1161-1176.

[3] T.J. Lu, A. Hess, M.F. Ashby, Sound absorption in metallic foams, Journal of Applied Physics, 85 (1999) 7528-7539.

[4] J.H. Wu, Z.P. Hu, H. Zhou, Sound absorbing property of porous metal materials with high temperature and high sound pressure by turbulence analogy, Journal of Applied Physics, 113 (2013) 194905.

[5] A. Hasan, B. Foss, S. Sagatun, Flow control of fluids through porous media, Appllied Mathematics and Computation, 219 (2012) 3323-3335.

[6] C. H. Bruneau, I. Mortazavi, Numerical modelling and passive flow control using porous media, Computers and Fluids, 37 (2008) 488-498.

[7] M.F. Ashby, T. Lu, Metal foams: a survey, Science in China Series B: Chemistry, 46 (2003) $521-532$.

[8] L. P. Lefebvre, J. Banhart, D. Dunand, Porous metals and metallic foams: current status and recent developments, Adv Eng Mater, 10 (2008) 775-787.

[9] T. Geyer, E. Sarradj, C. Fritzsche, Measurement of the noise generation at the trailing edge of porous airfoils, Experiments in Fluids, 48 (2010) 291-308. 
[10] T. Geyer, E. Sarradj, C. Fritzsche, Porous airfoils: noise reduction and boundary layer effects, International Journal of Aeroacoustics, 9 (2010) 787-820.

[11] T. Sueki, T. Takaishi, M. Ikeda, N. Arai, Application of porous material to reduce aerodynamic sound from bluff bodies, Fluid Dynanmics Reseaerch, 42 (2010) 015004.

[12] T. Sueki, A. Ikeda, T. Takaishi, T. Kurita, H. Yamada, Reduction of aerodynamic noise from high-speed pantograph using porous materials, Journal of Environmental Engineering, 5 (2010) 469-484.

[13] D.L. Sutliff, M.G. Jones, Low-speed fan noise attenuation from a foam-metal liner, Journal of aircraft, 46 (2009) 1381.

[14] D.L. Sutliff, M.G. Jones, T.C. Hartley, High-speed turbofan noise reduction using foammetal liner over-the-rotor, Journal of Aircraft, 50 (2013) 1491-1503.

[15] F. Paun, S. Gasser, L. Leylekian, Design of materials for noise reduction in aircraft engines, Aerosp Science and Technology, 7 (2003) 63-72.

[16] C. Xu, Y. Mao, Z. Hu, Tonal and broadband noise control of an axial-flow fan with metal foams: Design and experimental validation, Appled Acoustics, 127 (2017) 346-353.

[17] C. Xu, Y. Mao, Passive control of centrifugal fan noise by employing open-cell metal foam, Applied Acoustics, 103 (2016) 10-19.

[18] C. Xu, Y. Mao, Experimental investigation of metal foam for controlling centrifugal fan noise, Applied Acoustics, 104 (2016) 182-192.

[19] J. Jimenez, M. Uhlmann, A. Pinelli, G. Kawahara, Turbulent shear flow over active and passive porous surfaces, Journal of Fluid Mechanics, 442 (2001) 89-117.

[20] A.F. Tinetti, On the use of surface porosity to reduce wake-stator interaction noise, Virginia Polytechnic Institute and State University, 2001. 
[21] H. Lai, K.H. Luo, A conceptual study of cavity aeroacoustics control using porous media inserts, Flow, Turbulence and Combustion, 80 (2008) 375-391.

[22] M.S. Suzuki, T. Takaishi, T. Nakade, K, A numerical study on mechanism of aerodynamic noise reduction by porous material, The Sixteenth International Congress on Sound and Vibration, Krakow 5th-9th, July, 2009 1-6.

[23] C.H. Bruneau, L. Mortazavi, Passive control of the flow around a square cylinder using porous media, International Journal for Numerical Methods in Fluids, 46 (2004) 415-433.

[24] C.T. Hsu, P. Cheng, Thermal Dispersion In a Porous-Medium, International Journal of Heat and Mass Transfer, 33 (1990) 1587-1597.

[25] K. Vafai, Convective Flow And Heat-Transfer In Variable-Porosity Media, Journal of Fluid Mechanics, 147 (1984) 233-259.

[26] H. Darcy, Les fontaines publiques de la ville de Dijon: exposition et application, Victor Dalmont1856.

[27] S. Ergun, Fluid Flow Through Packed Columns, Chemical Engineering Progress, 48 (1952) 89-94.

[28] S. Bhattacharyya, A.K. Singh, Reduction in drag and vortex shedding frequency through porous sheath around a circular cylinder, International Journal for Numerical Methods in Fluids, 65 (2011) 683-698.

[29] H. Liu, J. Wei, Z. Qu, Prediction of aerodynamic noise reduction by using open-cell metal foam, Journal of Sound and Vibration, 331 (2012) 1483-1497.

[30] H. Liu, J.Wei, Z. Qu, The interaction of porous material coating with the near wake of bluff body, ASME Journal of Fluid Engieering, 136 (2014) 021302. 
[31] H. Naito, K. Fukagata, Numerical simulation of flow around a circular cylinder having porous surface, Physics of Fluids, 24 (2012).

[32] J. Schulze, J. Sesterhenn, Optimal distribution of porous media to reduce trailing edge noise, Computers and Fluids, 78 (2013) 41-53.

[33] Y. Bae, Y.J. Moon, Effect of passive porous surface on the trailing-edge noise, Physics of Fluids, 23 (2011) 126101.

[34] S. Krishnan, J.Y. Murthy, S.V. Garimella, Direct simulation of transport in open-cell metal foam, ASME Journal of Heat transfer, 128 (2006) 793-799.

[35] K. Boomsma, D. Poulikakos, Y. Ventikos, Simulations of flow through open cell metal foams using an idealized periodic cell structure, International Journal of Heat Fluid Flow, 24 (2003) 825-834.

[36] S. Patankar, C. Liu, E. Sparrow, Fully developed flow and heat transfer in ducts having streamwise-periodic variations of cross-sectional area, ASME Journal of Heat Transfer, 99 (1977) 180-186.

[37] Z. Hu, C.L. Morfey, N.D. Sandham, Aeroacoustics of wall-bounded turbulent flows, AIAA Journal, 40 (2002) 465-473.

[38] Z. Hu, C.L. Morfey, N.D. Sandham, Sound radiation in turbulent channel flows, Journal of Fluid Mechanics, 475 (2003) 269-302.

[39] F. Kuwahara, I. Yamane, A. Nakayama, Large eddy simulation of turbulent flow in porous media, International Communications in Heat and Mass Transfer, 33 (2006) 411-418.

[40] S.V. Patankar, D.B. Spalding, A calculation procedure for heat, mass and momentum transfer in three-dimensional parabolic flows, International Journal of Heat and Mass Transfer, 15 (1972) 1787-1806. 
[41] C. Wagner, T. Huttl, P. Sagaut, Large-eddy simulation for acoustics, Cambridge University Press, Cambridge, 2006.

[42] N.J. Georgiadis, D.P. Rizzetta, C. Fureby, Large-eddy simulation: current capabilities, recommended practices, and future research, AIAA Journal, 48 (2010) 1772-1784.

[43] A. Bhattacharya, V. Calmidi, R. Mahajan, Thermophysical properties of high porosity metal foams, International Journal of Heat and Mass Transfer, 45 (2002) 1017-1031.

[44] M. Lacroix, P. Nguyen, D. Schweich, C.P. Huu, S. Savin-Poncet, D. Edouard, Pressure drop measurements and modeling on SiC foams, Chemical Engineering Science, 62 (2007) 32593267.

[45] L. Giani, G. Groppi, E. Tronconi, Mass-transfer characterization of metallic foams as supports for structured catalysts, Industrial and Engieering Chemistry Research, 44 (2005) 49935002.

[46] J. Richardson, Y. Peng, D. Remue, Properties of ceramic foam catalyst supports: pressure drop, Applied Catalysis A: General, 204 (2000) 19-32.

[47] J.L. Florenciano, P. Bruel, LES fluid-solid coupled calculations for the assessment of heat transfer coefficient correlations over multi-perforated walls, Aerospace Science and Technology, 53 (2016) 61-73.

[48] M. Kompenhans, E. Ferrer, M. Chavez, E. Valero, Numerical study of three dimensional acoustic resonances in open cavities at high Reynolds numbers, Aerospace Science and Technology, 45 (2015) 501-511.

[49] M.J. Lighthill, On sound generated aerodynamically. I. general theory, Proceedings of the Royal Society of London, Series A, Mathematical and Physical Sciences, 211 (1952) 564-587. 
[50] N. Curle, The influence of solid boundaries upon aerodynamic sound, Proceedings of the Royal Society of London Series A, Mathematical and Physical Sciences, 231 (1955) 505-514. 